\title{
Grain-Boundary Sliding in AZ31 Magnesium Alloys at Room Temperature to $523 \mathrm{~K}$
}

\author{
J. Koike, R. Ohyama, T. Kobayashi, M. Suzuki and K. Maruyama \\ Department of Materials Science, Tohoku University, Sendai 980-8579, Japan
}

Rolled sheets of AZ31 Mg alloys were subjected to tensile testing at temperatures ranging from room temperature to $523 \mathrm{~K}$. The occurrence of grain-boundary sliding (GBS) at room temperature was demonstrated by the displacement of scribed lines across grain boundaries of deformed samples. Surface relief of deformed samples was measured by use of a scanning laser microscope. GBS strain was calculated from the measured surface step height, and its temperature dependence was analyzed by a Dorn-type constitutive equation. GBS above $423 \mathrm{~K}$ was found to be pure GBS that was activated by resolved applied shear stress acting on grain boundaries. The activation energy for GBS was found to be $80 \mathrm{~kJ} / \mathrm{mol}$, which is in agreement with the activation energy for grain boundary diffusion. Meanwhile, GBS below $373 \mathrm{~K}$ was found to be slipinduced GBS, and its extent was found to be significantly greater than that expected from extrapolation of high-temperature values. The slipinduced GBS is considered to occur by plastic compatibility conditions in the presence of plastic strain anisotropy and by absorption and dissociation of lattice dislocations at grain boundaries.

(Received November 11, 2002; Accepted March 3, 2003)

Keywords: magnesium, mechanical property, grain-boundary sliding, room temperature

\section{Introduction}

Plastic deformation of $\mathrm{Mg}$ alloys at ambient temperature is considered to be dominated by dislocation slip on hep basal planes. ${ }^{1-4)}$ However, basal dislocation slip can provide only two independent slip systems, which is not sufficient for satisfying the von-Mises criterion for homogeneous deformation. Despite the presumably small number of independent slip systems, many Mg alloys exhibit a reasonable ductility of more than $10 \%$ in tensile elongation to failure. ${ }^{5)}$ Moreover, some fine-grained $\mathrm{Mg}$ alloys exhibit a tensile elongation of $47 \%$ at room temperature at a strain rate of $1 \times 10^{-3} \mathrm{~s}^{-1}{ }^{6)}$ In order to understand the origin of large tensile ductility in these fine-grained $\mathrm{Mg}$ alloys, we investigated dislocation activity and found that $40 \%$ of dislocation segments are of non-basal type. ${ }^{7)}$ Such a remarkable activity of non-basal segments was attributed to compatibility stress at grain boundaries. Fine grain size was then considered effective for enhancing the compatibility effect.

Another mechanism that can be enhanced by grain refinement is grain-boundary sliding. The possibility of enhanced grain-boundary sliding (GBS) in Mg alloys below ambient temperature was reported by Hauser et al. ${ }^{8,9)}$ In their paper, GBS was termed grain-boundary shearing, and the possibility of its operation was examined in pure $\mathrm{Mg}$ $(99.97 \%)$ at room temperature and at $78 \mathrm{~K} .^{9)}$ Displacement of scribed lines at grain boundaries was observed at both temperatures and is considered to be an evidence for the occurrence of grain-boundary shearing. However, in their experiment, grain-boundary shearing was often accompanied by grain-boundary fracture. Therefore, their report fails to clarify whether GBS actually took place at room temperature and $78 \mathrm{~K}$.

Gifkins and Langdon ${ }^{10)}$ carefully examined the possibility of GBS at room temperature in pure $\mathrm{Mg}$ and $\mathrm{Mg}$ alloys having grain sizes of 100 to $300 \mu \mathrm{m}$. They observed the offset of scribed lines and of interference fringes after deformation. In most cases, the scribed lines were continuously bent near grain boundaries without any offset. Some offset regions could be found, but they became continuous with increasing strain. At strain exceeding $0.2 \%$, the strain dependence of mean step height across grain boundaries was found to saturate at a mean step height of about $0.1 \mu \mathrm{m}$, indicating that no GBS occurred in this region of strain. In contrast, above $398 \mathrm{~K}$, mean step height showed strong dependence of strain and temperature. From the difference in step formation behavior at room temperature and at $398 \mathrm{~K}$, they concluded that GBS does not occur in $\mathrm{Mg}$ and its alloys at room temperature and that the step formation was due to localized shear deformation of different magnitudes between adjacent grains. This phenomenon has been known as "zone sliding," but is not GBS.

Meanwhile, other authors have reported that GBS can occur at room temperature in nanocrystalline $\mathrm{Mg},{ }^{11)}$ as well as in other nanocrystalline metals such as $\mathrm{Ni}^{12)}$ and $\mathrm{Au}{ }^{13)}$ Hwang et al. ${ }^{11)}$ investigated strain-rate sensitivity of nanograined $\mathrm{Mg}$ at room temperature. Their samples were prepared by ball milling and cold pressing to attain a grain size of $45 \mathrm{~nm}$. Strain rate jump tests indicate that the stress exponent value, $n$, is 1.65 with a threshold stress of $125 \mathrm{MPa}$. They claimed that the $n$ value is close to a predicted value of 2 for GBS accommodated by the dislocation glide mechanism.

The tendency of GBS increasing with decreasing grain size has gained general acceptance. ${ }^{14)}$ Many commercially available $\mathrm{Mg}$ alloys can be reduced in grain size to less than $10 \mu \mathrm{m}$. However, whether or not GBS can occur in finegrained Mg alloys remains unknown. Since GBS can make an important contribution to the ductility of fine-grained alloys, we investigated the possibility of GBS at room temperature and compared the results with observations of GBS at elevated temperatures.

\section{Experimental Procedure}

A sheet of AZ31B-O (Mg-3Al-1Zn-0.3Mn in mass\%) had been rolled at $433 \mathrm{~K}$ and annealed at $618 \mathrm{~K}$ for 2 hours. Average grain size was $8 \mu \mathrm{m}$. Tensile specimens were machined to have a gage section of $10 \mathrm{~mm}$ length, $3 \mathrm{~mm}$ width, and $1.2 \mathrm{~mm}$ thickness. Tensile tests were performed in 
air to various strains up to an elongation of $17 \%$ at $300 \mathrm{~K}$. At elevated temperatures of $373,423,473$, and $523 \mathrm{~K}$, tensile tests were performed to a fixed elongation value of $10 \%$. For all the tests, initial strain rate was set constant at $8.3 \times 10^{-4} \mathrm{~s}^{-1}$. Stress was applied parallel to the rolling direction.

Before tensile tests, sample surface was electrochemically polished at $238 \mathrm{~K}$ at an applied voltage of $55 \mathrm{~V}$ using a solution of $60 \mathrm{ml}$ ethanol, $4 \mathrm{ml}$ 1,2-butoxyethatnol, and $1 \mathrm{ml}$ hydrochloric acid. Surface relief formed after deformation was observed using a scanning laser microscope and a conventional optical microscope. A height profile was obtained along a given length of a line in a laser-scanned image. The magnitude of GBS was calculated by measuring surface step height at grain boundaries according to the following equation proposed by Bell and Langdon. ${ }^{15)}$

GBS strain can be calculated from the relation

$$
\begin{aligned}
\varepsilon_{\mathrm{GBS}} & =\frac{u}{L} \\
& =k \frac{v}{L} .
\end{aligned}
$$

where, $u$ is a component of GBS displacement parallel to the tensile direction, and $v$ is a component of GBS displacement perpendicular to the sample surface as well as to the tensile direction. In many cases, $u$ is difficult to measure, and $v$ is measured instead. The constant $k$ is a geometrical factor for converting $u$ to $v$. Based on theoretical and experimental evaluation, Bell and Langdon ${ }^{15)}$ have determined $k$ to be 1.4 for annealed samples and 1.1 for mechanically or chemically polished samples. Since our samples were electrochemically polished, the $k$ value of 1.1 was used to calculate GBS strain. In the present work, the sum of the all step heights at grainboundary locations along the scanned line was taken as $v$, and the scanned length was taken as $L$ in eq. (2). In order to increase the reliability in determining GBS strain, step height measurement was performed on 10 different lines in one sample. The obtained values were averaged, and standard deviation was taken as measurement error.

Separate experiments were also performed to show a solid evidence of grain-boundary sliding at room temperature. Electrochemically polished samples were placed in a focused ion beam (FIB) machine. Line patterns were drawn by locally sputtering a sample region using a focused $\mathrm{Ga}$ ion beam accelerated at $30 \mathrm{kV}$. The targeted dimension of the line was $100 \mu \mathrm{m}$ in length, $0.1 \mu \mathrm{m}$ in width, and $0.2 \mu \mathrm{m}$ in depth. Then the samples were tensile tested to an elongation of $10 \%$ at room temperature at an initial strain rate of $1 \times 10^{-3} \mathrm{~s}^{-1}$. The surface of the deformed samples was observed by the FIB machine in order to examine the displacement of the lines at grain boundaries. The FIB machine was capable of not only imaging the lines but also imaging grain boundaries clearly even in the polished samples.

\section{Results}

Nominal stress and strain curves are shown in Fig. 1 for various temperatures. At room temperature, $0.2 \%$ proof stress and ultimate tensile stress are 190 and $295 \mathrm{MPa}$, respectively. Fracture strain is $20 \%$. When deformation temperature is

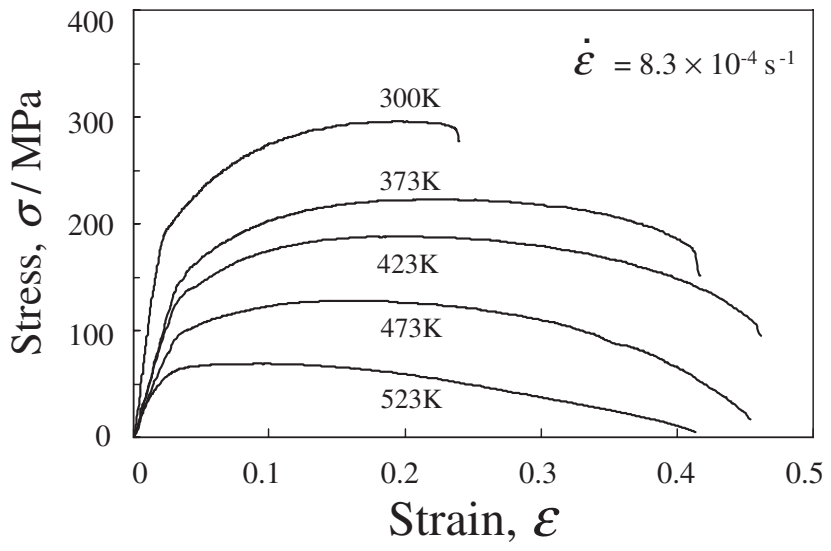

Fig. 1 Nominal stress-strain curves of AZ31B-O Mg alloys at a strain rate of $8.3 \times 10^{-4} \mathrm{~s}^{-1}$.

increased beyond $373 \mathrm{~K}$, fracture strain increases to more than $40 \%$.

Figure 2 show an FIB image of deformed samples at room temperature to a strain of $10 \%$. Tensile axis is along the horizontal direction of the figure. Most of the grain boundaries can be clearly recognized. White lines in the vertical direction are the lines drawn by the FIB machine. Curved white lines within each grain are surface steps formed by dislocation slip. It can be seen that originally straight lines are abruptly shifted at eight grain-boundary locations, indicated by arrows. A magnified image is shown on the right to show the line shift more clearly. This location exhibits the largest shift among the eight locations. The magnitude of the shift is approximately $0.3 \mu \mathrm{m}$ in the horizontal direction. Considering the diameter of the corresponding grain being approximately $12 \mu \mathrm{m}$, the GBS displacement of $0.3 \mu \mathrm{m}$ yields a GBS strain of $2.5 \%$, one fourth of the total strain of $10 \%$. The average magnitude of the shift at the eight locations is found to be $0.18 \mu \mathrm{m}$. This value can be compared later with the magnitude of vertical shift measured by a scanning laser microscope. Notice that there are many line segments that are not shifted at grain boundaries. These results indicate that the occurrence and magnitude of GBS is dependent on the grain boundary structure.

Figure 3 shows surface morphology taken by an optical microscope before and after deformation to $10 \%$ at 300,423 , and $523 \mathrm{~K}$. At all temperatures, surface relief is observed after deformation and locations of grain boundaries can be clearly recognized in most grains. As deformation temperature is increased to $523 \mathrm{~K}$, image contrast of grain boundaries becomes sharp. Steps are observed along nearly all grain boundaries.

Figure 4 shows the variation of surface relief along a given line in scanning laser micrographs after deformation to 0.1 . The vertical scale in the figure is magnified by six times with respect to the horizontal scale. Vertical dotted lines in the figure indicate the locations of grain boundaries determined by matching the surface-relief profile with the corresponding image of scanning laser microscopy. Before deformation, the surface was flat, within $+/-0.013 \mu \mathrm{m}$ in vertical height. After deformation at room temperature, the surface becomes 

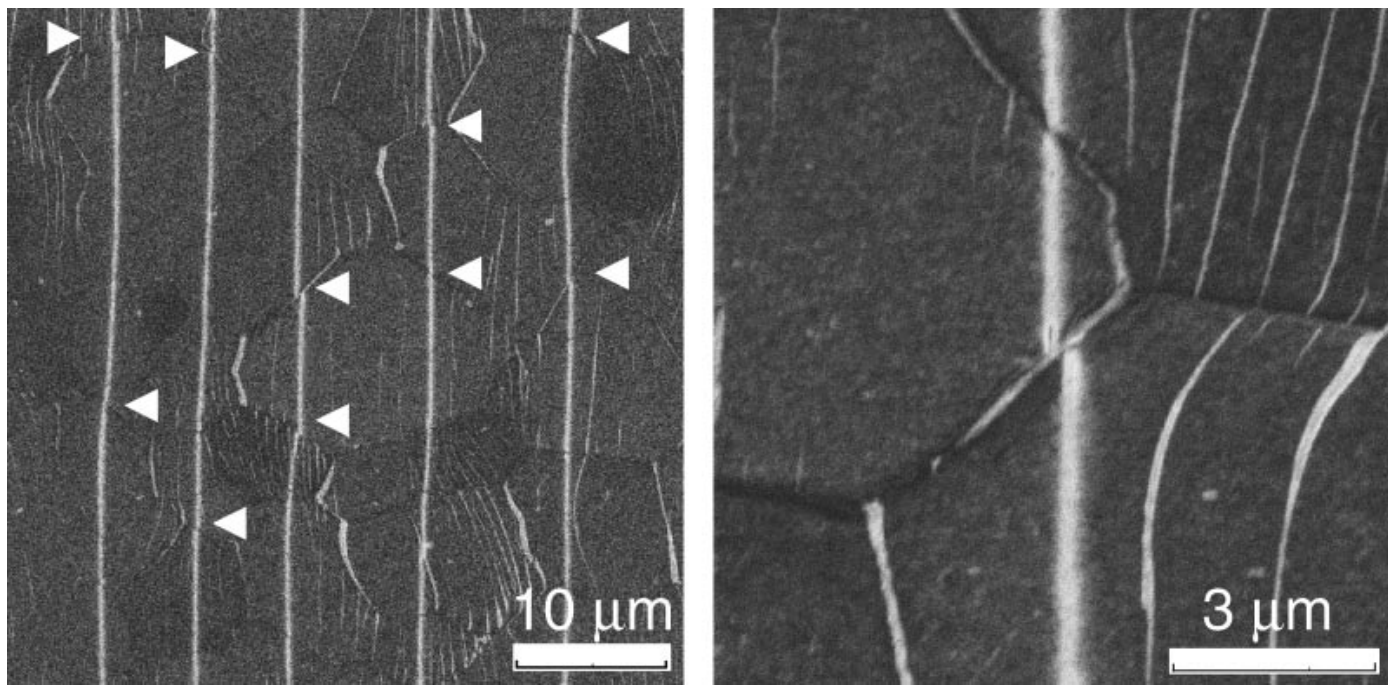

Fig. 2 Focused ion beam (FIB) images of a sample deformed at room temperature to $10 \%$. Vertical lines were drawn by the FIB machine. A figure on the right is a magnified image of the image on the left. Tensile axis is along the horizontal direction of the figure.
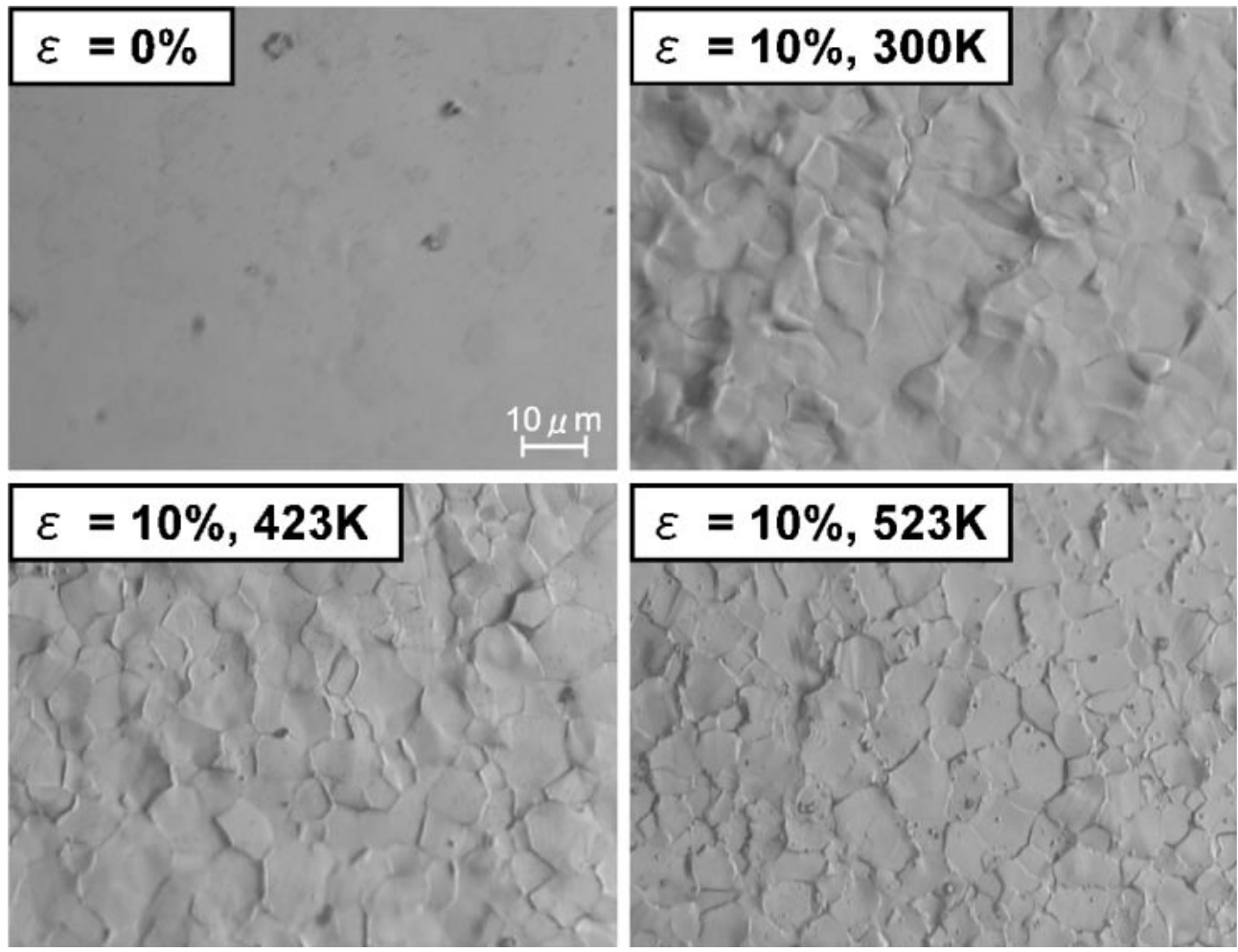

Fig. 3 Optical micrographs of the surface before deformation and after deformation to $10 \%$ at 300,423 , and $523 \mathrm{~K}$.

severely undulated probably caused by inhomogeneity in plastic deformation due to preferential basal slipping of dislocations. An undulation segment may be a structural unit in which deformation occurs in a similar anisotropic manner. The occurrence of GBS is seen as an abrupt change in height at some grain boundaries. The average step height is $0.15 \mu \mathrm{m}$. According to eqs. (1) and (2), this value can be converted by multiplying the $k$ factor of 1.1 to a horizontal shift of $0.165 \mu \mathrm{m}$. Notice that the converted horizontal shift is in good agreement with the average value of $0.18 \mu \mathrm{m}$ estimated from the line shift in the FIB image. As deformation temperature increases, the extent of undulation decreases and surface steps at grain boundaries become a dominant feature in the surface-relief profile. At $523 \mathrm{~K}$, the surface is perfectly flat except at grain boundaries. Some minor height changes are observed in the grain interior, but they are due to etch pits formed during electrochemical polishing. The diminishment of undulation with temperature is probably caused by the activation of non-basal slip systems at elevated temperatures, leading to increased homogeneity in intragranular deformation. 


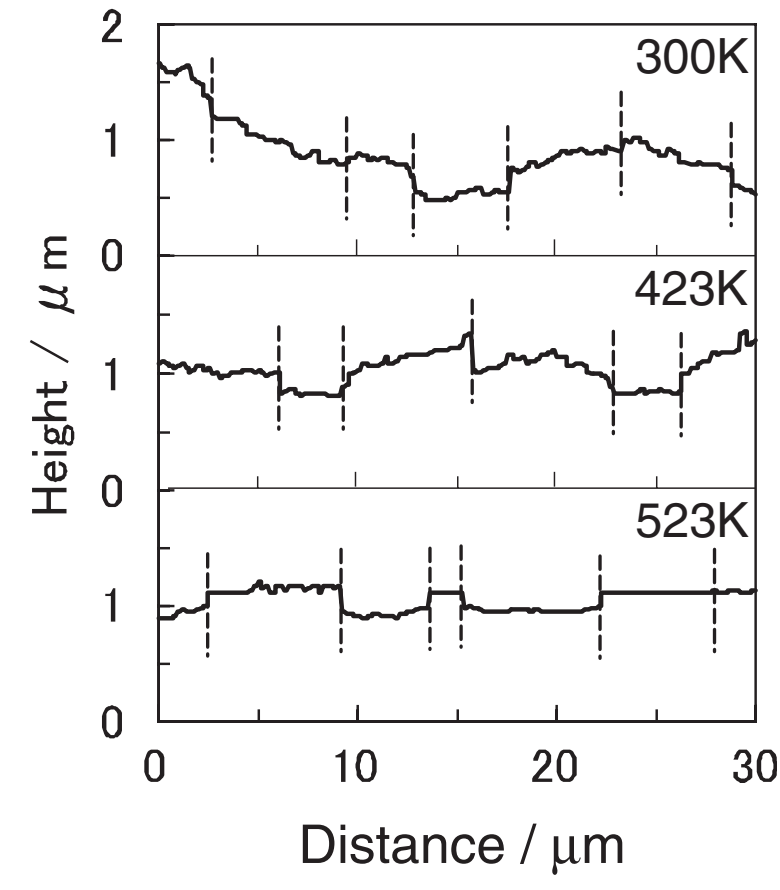

Fig. 4 Surface-relief profile obtained by a scanning-laser microscope along a given line of the sample surface after deformation to 0.1 at 300 , 423 , and $523 \mathrm{~K}$

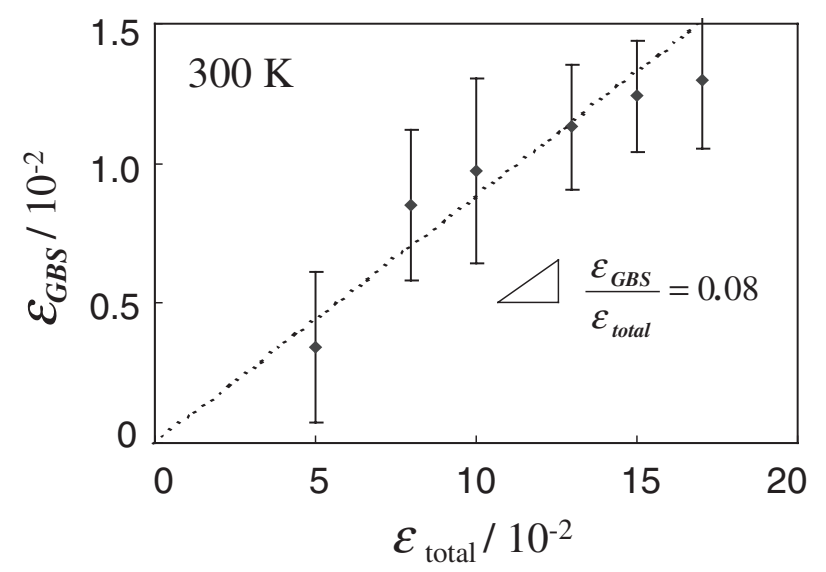

Fig. 5 Variation of GBS strain as a function of total strain at $300 \mathrm{~K}$.

In order to calculate GBS strain from eq. (2), total step height is measured for the given length of the line in the surface-relief profiles. Figure 5 shows GBS strain as a function of total strain measured at room temperature. As seen from the figure, GBS strain increases almost linearly with total strain. The ratio of GBS strain to total strain is obtained from the slope, and is approximately $8 \%$ at room temperature. Figure 6 shows GBS strain at a total plastic strain of 0.1 as a function of deformation temperature. As temperature increases to $423 \mathrm{~K}$, GBS strain gradually increases. Above $423 \mathrm{~K}$, GBS strain increases sharply with temperature. At $523 \mathrm{~K}$, the ratio of GBS strain to total plastic strain becomes as high as $27 \%$.

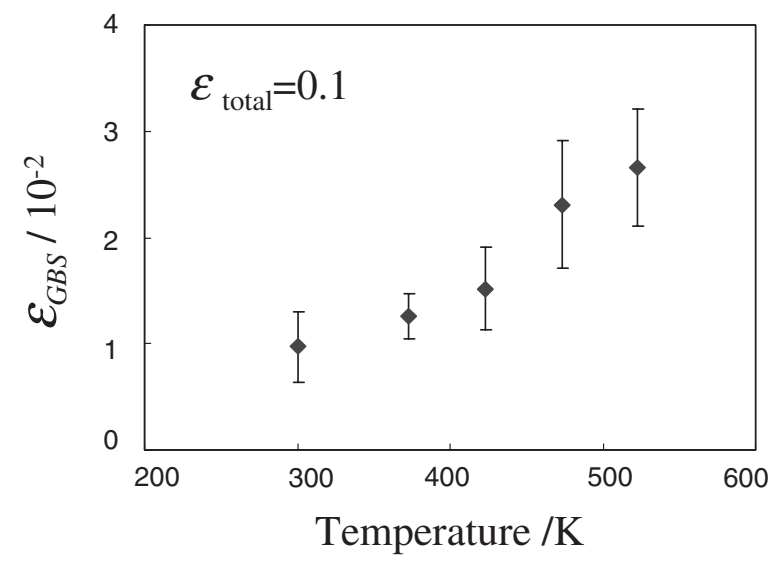

Fig. 6 Temperature dependence of GBS strain at a total strain of 0.1.

\section{Discussion}

\subsection{Temperature dependence of GBS}

The present work demonstrates that GBS occurs at room temperature as well as at elevated temperatures up to $523 \mathrm{~K}$. The characteristics of GBS at room temperature are revealed by comparison with GBS at elevated temperature, as follows.

GBS strain rate can be expressed by a Dorn-type equation as a function of applied stress and temperature, as follows, ${ }^{16,17)}$

$$
\dot{\varepsilon}_{\mathrm{GBS}}=A\left(\frac{G b}{k T}\right)\left(\frac{b}{d}\right)^{p}\left(\frac{\sigma}{G}\right)^{n} \exp \left(-\frac{E}{R T}\right) .
$$

Here, $A, p$, and $n$ are constants; $G$ is shear modulus; $b$ is the Burgers vector; $d$ is grain size; $\sigma$ is applied stress; $E$ is activation energy; and $R T$ and $k T$ have their usual meanings. As shown in Fig. 5, within experimental accuracy, a linear relation can be assumed between GBS strain and total strain as follows,

$$
\frac{\varepsilon_{\mathrm{GBS}}}{\varepsilon_{\text {total }}} \approx \text { const. }
$$

Although the present work is carried out at constant crosshead speed, total strain rate is approximated to be constant within the experimental range of strain. Thus, GBS strain rate is expressed as

$$
\dot{\varepsilon}_{\mathrm{GBS}} \approx \frac{\varepsilon_{\mathrm{GBS}}}{t} \approx \frac{\varepsilon_{\mathrm{GBS}}}{\varepsilon_{\text {total }} / \dot{\varepsilon}_{\text {total }}}
$$

Under constant total strain rate, GBS strain rate also becomes constant, because of the relation expressed by eq. (4). Therefore, by modifying eq. (3), the temperature dependence of GBS strain can be evaluated for a fixed value of total strain under a constant strain rate:

$$
\varepsilon_{\mathrm{GBS}}=A^{\prime}\left(\frac{G b}{k T}\right)\left(\frac{\sigma}{G}\right)^{n} \exp \left(-\frac{E}{R T}\right),
$$

where

$$
A^{\prime}=A\left(\frac{b}{d}\right)^{p} \frac{\varepsilon_{\text {total }}}{\dot{\varepsilon}_{\text {total }}} .
$$

This equation is further modified to 


$$
\varepsilon_{\mathrm{GBS}}=\left(\frac{k T}{G b}\right)\left(\frac{\sigma}{G}\right)^{-n}=A^{\prime} \exp \left(-\frac{E}{R T}\right) .
$$

An Arrhenius type plot of eq. (7) yields apparent activation energy for GBS sliding. To create the plot, applied stress at plastic strain of 0.1 at a strain rate of $8.3 \times 10^{-4} \mathrm{~s}^{-1}$ are taken from Fig. 1 and inserted into eq. (7). Stress exponent, $n$, for GBS is assumed to be 4, as has been experimentally determined for various metals. ${ }^{18,19)}$ It should be mentioned that the stress exponent is not the one for ordinary creep deformation. The temperature dependence of $G$ is also considered: ${ }^{20)}$

$$
G=1.92 \times 10^{-4}-8.6 T \text { in } \mathrm{MPa} .
$$

Figure 7 shows an Arrhenius plot of eq. (7). The data points in the figure from left to right correspond to deformation temperatures of 523, 473, 423, 373, and $300 \mathrm{~K}$, respectively. A straight line can be drawn through the three data points at temperatures above $423 \mathrm{~K}$, indicating that a same mechanism is responsible for GBS. The slope of the line gives an activation energy of $80 \mathrm{~kJ} / \mathrm{mol}$. This value is in good agreement with the activation energy for grainboundary diffusion of $\mathrm{Mg}^{21)}$ Therefore, the GBS mechanism above $423 \mathrm{~K}$ is rate-controlled by grain-boundary diffusion. In contrast, the data points for $373 \mathrm{~K}$ and $300 \mathrm{~K}$ deviate to larger values from the line extrapolated from high temperatures. The deviation suggests that the stress exponent of 4 may not be valid below $373 \mathrm{~K}$ possibly because of a different rate-controlling mechanism for GBS. A weak temperature dependence also suggests that the activation energy is smaller below $373 \mathrm{~K}$ than those above $423 \mathrm{~K}$. The temperature dependence of GBS may be considered in a similar manner to that of dislocation glide. In the case of dislocation glide, deformation mechanisms change, with decreasing temperature, from power-law creep with a constant stress exponent, to power-law breakdown with a much higher value of stress exponent, and to dislocation plasticity. In this regard, it would be reasonable to assume that the actual value of stress exponent is larger than 4 below $373 \mathrm{~K}$. If a larger stress exponent were used for the two data points below $373 \mathrm{~K}$, the data points would be positioned at much higher locations in the figure, leading to a larger deviation. Therefore, the deviation is not due to artifact caused by the choice of

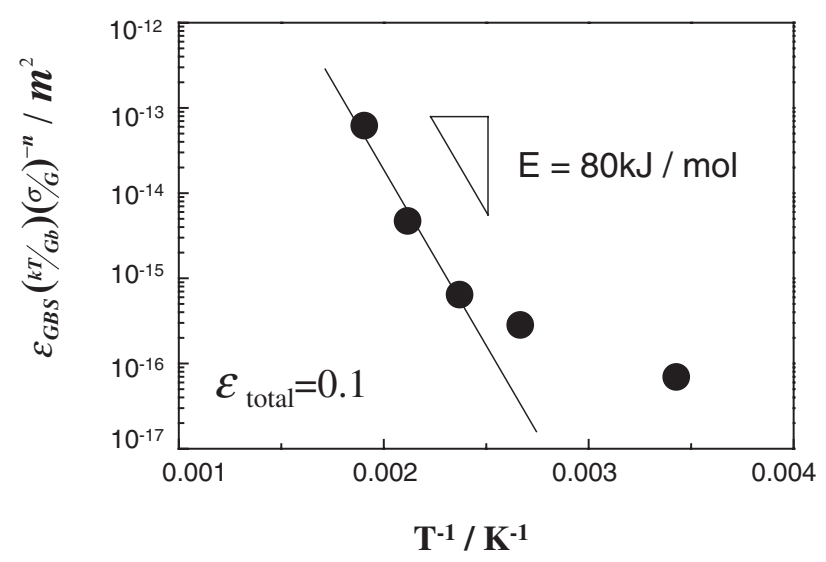

Fig. 7 Arrhenius plot of temperature dependence of GBS strain. parameters. Rather it represents the enhancement of GBS. The magnitude of deviation is found to be 3 times at $373 \mathrm{~K}$ and 40 times at $300 \mathrm{~K}$ in comparison with the extrapolated values. We consider GBS above $423 \mathrm{~K}$ to be "pure" GBS, whereas GBS below $373 \mathrm{~K}$ to be "slip-induced" GBS. ${ }^{22)}$

\subsection{Mechanism of slip-induced GBS}

Mussot et al. $^{23)}$ demonstrated, both theoretically and experimentally, that GBS could be enhanced by the compatibility requirement of anisotropic plastic strain at grain boundaries. Since plastic anisotropy of $\mathrm{Mg}$ alloys is significantly high at room temperature, slip-induced GBS can be expected in Mg alloys. The slip-induced GBS was interpreted by use of three strain tensors, $a_{i j}, b_{i j}$, and $c_{i j}$, of bicrystal shown in Fig. 8, where a grain boundary includes $x_{1}$ and $x_{3}$ axes and is placed at $x_{2}=0$. One crystal, denoted L, lies within the region $x_{2}<0$ and another, denoted $\mathrm{R}$, lies within the region $x_{2}>0$. Plastic strain in the $\mathrm{L}$ crystal is described by $a_{i j}$, and that in the $\mathrm{R}$ crystal it is $a_{i j}+b_{i j}$; therefore, $b_{i j}$ corresponds to the difference in plastic strain between L and R. If GBS is not allowed, as is usually the case, these two strain tensors are sufficient for attaining the compatibility condition. In order to describe GBS, Mussot et $a l$. introduced $c_{i j}$ as a strain tensor at the grain boundary plane. Note that $c_{22}=0$ for not allowing decohesion at the boundary. Therefore, total strain is given by

$$
\begin{aligned}
& \varepsilon_{i j}\left(x_{1}, x_{2}, x_{3}\right)=a_{i j}\left(x_{1}, x_{2}, x_{3}\right) \\
& \quad+b_{i j}\left(x_{1}, x_{2}, x_{3}\right) \cdot Y\left(x_{2}\right)+c_{i j} \cdot \delta\left(x_{2}\right)
\end{aligned}
$$

where $Y\left(x_{2}\right)$ is a Heaviside step function that assumes 0 for $x_{2}<0$ and 1 for $x_{2}>0$, and $\delta\left(x_{2}\right)$ is a Dirac delta function. The compatibility conditions are given by ${ }^{24)}$

$$
\varepsilon_{i j k} \varepsilon_{l m n} \varepsilon_{j n, k m}=0
$$

where $\varepsilon_{i j k}$ and $\varepsilon_{l m n}$ are the permutation tensors and $\varepsilon_{j n, k m}$ is the second derivative of the strain tensor. The compatibility conditions are evaluated at three regions; $x_{2}<0, x_{2}=0$, and $x_{2}>0$, and are reduced to the following conditions.

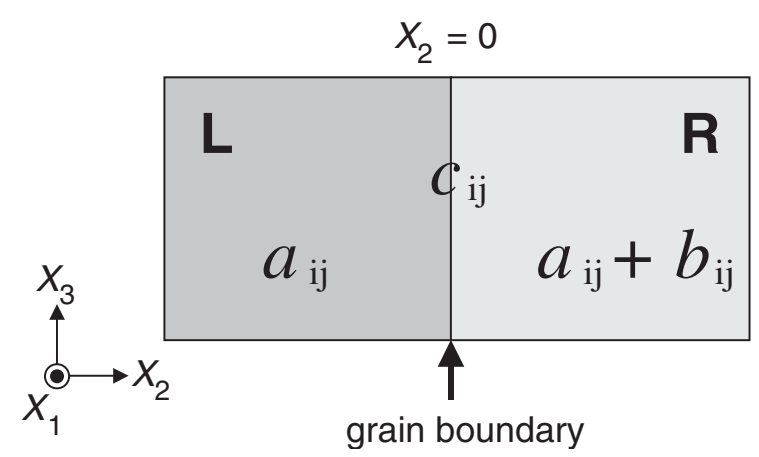

Fig. 8 Strain tensors in bicrystal bonded at a grain boundary in an $x_{1}-x_{3}$ plane located at $x_{2}=0$. Crystals on the left and the right are denoted $\mathrm{L}$ and $\mathrm{R}$, respectively. Strain tensor in $\mathrm{L}$ is given by $a_{i j}$, and for $\mathrm{R}$ an additional tensor term $b_{i j}$ is added in order to express a different magnitude of strain attributable to relative crystallographic orientation between L and R. GBS strain is given by $c_{i j}$ and is localized within the grain boundary. (after Mussot et al. ${ }^{23)}$ ) 


$$
\begin{aligned}
& \varepsilon_{i j k} \varepsilon_{l m n} a_{j n, k m}=0 \\
& \varepsilon_{i j k} \varepsilon_{l m n} b_{j n, k m}=0 \\
& c_{11}=c_{13}=c_{33}=0 \\
& c_{12,1}=\left.\frac{1}{2} b_{11}\right|_{x_{2}=0} \\
& c_{23,3}=\left.\frac{1}{2} b_{33}\right|_{x_{2}=0} \\
& c_{12,3}+c_{23,1}=\left.b_{13}\right|_{x_{2}=0}
\end{aligned}
$$

Equations (11a) and (11b) yield ordinary compatibility conditions without GBS, and the other equations are unique for GBS. The anisotropic strain components of $b_{11}, b_{33}$, and $b_{13}$ at the boundary position are shown to give rise to strain variation concentrated at the boundary. In order to verify the relation expressed by eq. (11e), Mussot et al. experimentally confirmed the variation of $C_{23}$ along the $x_{3}$ direction of the grain boundary, and found that $b_{33}$ is much larger than the value calculated on the sole basis of plastic anisotropy. They considered the enhancement of $b_{33}$ and GBS to be interrelated, in order to satisfy overall compatibility conditions at grain boundaries.

In the case of $\mathrm{Mg}$, as clearly shown by Fig. 4, intragranular deformation is homogeneous at $523 \mathrm{~K}$, as indicated by the flatness of grain surface with occasional interruption of grainboundary steps. This homogeneous deformation is due to the activity of multiple slip systems and to the ease of dislocation climbing at $523 \mathrm{~K}$. As temperature decreases, intragranular deformation becomes increasingly inhomogeneous, as indicated by severe undulation of the sample surface, especially at room temperature. This inhomogeneous deformation is caused by preferential dislocation slip on basal planes, as in the case of $\mathrm{Zn}^{22)}$ On the basis of these results, the temperature dependence of GBS in the $\mathrm{Mg}$ alloys can be interpreted as follows. At high temperatures, no $b_{i j}$ component arises, because deformation is homogeneous. Thus, no slip-induced GBS is expected. The observed GBS is considered pure GBS that occurs under applied resolved shear stress acting on grain boundary planes. In contrast, at low temperatures, plastic anisotropy and deformation inhomogeneity bring about non-zero values of $b_{i j}$ components, leading to the occurrence of slip-induced GBS in addition to pure GBS.

Other evidence for slip-induced GBS was reported in $\mathrm{Zn}$ bicrystal deformed at room temperature and was interpreted in a different way. Valiev et al. ${ }^{22)}$ prepared a bicrystal sample in which basal planes of each grain are aligned perpendicular to each other. A grain boundary was formed at 45 degrees from these basal planes. They measured GBS strain at room temperature under two different tensile loading conditions. In the first condition, the tensile axis was set parallel to the basal planes of one grain and perpendicular to the basal planes of another grain. Whereas in the second condition, the tensile axis was tilted from the former case by 15 degrees. Since $\mathrm{Zn}$ is deformed by basal dislocation slip at room temperature, the first case does not induce dislocation slip within the grains, but the second case does. Using these samples, they then showed that in comparison with the first case, GBS rate of the second case was drastically enhanced by a factor of more than 50 times. The enhanced GBS was attributed to trapped lattice dislocations in grain boundaries that were dissociated to multiple displacement shift complete (DSC) dislocations. They pointed out that one of the dissociated components has Burgers vectors that are not parallel to the boundary planes and can climb out from the pile-up atmosphere. Thus, these dislocations were considered to be less susceptible to pile-up caused by GBS and to be more mobile than the intrinsic dislocations for pure GBS. ${ }^{22,25)}$

We should mention that the dislocation mechanism proposed by Valiev et al. and the compatibility mechanism proposed by Mussot et al. are focused on different aspects of the phenomenon and are not contradictory. We consider that both mechanisms contribute to the slip-induced GBS of the $\mathrm{Mg}$ alloys at room temperature.

A further question arises with regard to the accommodation mechanism of slip-induced GBS. A closer look at the relation between GBS strain and total strain in Fig. 5 may suggest a trend of GBS strain rate lowering with increasing total strain. This trend is known as slide hardening, and various mechanisms of slide hardening have been proposed. One possibility is that intersecting lattice dislocations or subboundaries interfere with dislocation slip in grain boundaries. $^{26,27)}$ Another possibility is pile-up of lattice dislocations at grain boundaries. ${ }^{28)}$ Slide hardening in $\mathrm{Mg}$ at high temperature may be effectively accommodated by grainboundary diffusion creep or by dislocation creep. Meanwhile, at low temperature, dislocation emission to the grain interior would be a dominant mechanism. In our recent work on the deformation mechanisms of fine-grained $\mathrm{Mg}$ alloys, nonbasal dislocation slip was found to occur easily. ${ }^{7)}$ However, the non-basal dislocation segments were formed mainly by cross slipping of screw dislocations from the basal planes. Since basal and non-basal segments are of $\boldsymbol{a}$ type dislocations, dislocation emission alone may fail to attain effective accommodation. Twinning may act as an additional accommodation mechanism. However, the role of twinning on deformation processes seems complicated, with various functions; i.e., (1) a complementary deformation mechanism that provides additional independent slip systems, ${ }^{2)}$ (2) formation of recovered regions, ${ }^{7,29,30)}$ (3) a source of work hardening by microstructure refinement as well as blocking of mobile dislocations, ${ }^{31)}$ and (4) a source of stress concentration and subsequent failure. Here, functions (1) and (2) can enhance ductility, whereas functions (3) and (4) reduce ductility. At the moment, however, the exact roles of twins in deformation and failure are not clearly delineated. Detailed investigation must be carried out before we come to understand the contribution of various types of twins to deformation and failure of $\mathrm{Mg}$ alloys.

\section{Summary}

AZ31 Mg alloys were subjected to tensile testing at a strain rate of $8.3 \times 10^{-4} \mathrm{~s}^{-1}$ at temperatures ranging from room temperature to $523 \mathrm{~K}$. Elongation to failure was $21 \%$ at room temperature and more than $40 \%$ at temperatures above $373 \mathrm{~K}$. FIB observation of deformed samples at room temperature indicates the displacement of scribed lines across grain boundaries, demonstrating the occurrence of GBS. Charac- 
teristic features of GBS were further investigated by measuring the surface step profile by use of a scanning laser microscope. GBS strain at room temperature was found to increase linearly with total strain, up to $15 \%$. The temperature dependence of GBS strain at a fixed total strain of $10 \%$ revealed that GBS above $423 \mathrm{~K}$ is ordinary "pure" GBS with an activation energy of $80 \mathrm{~kJ} / \mathrm{mol}$, which is close to the activation energy of grain boundary diffusion. In contrast, the extent of GBS below $373 \mathrm{~K}$ was found to be greater than the values extrapolated from the GBS data obtained at higher temperatures. In particular, GBS at room temperature is enhanced to 40 times the value extrapolated from the hightemperature data. Meanwhile, presumably inhomogeneous deformation at room temperature causes substantial surface undulation within a length of several grains. The undulation was found to decrease with increasing temperature. The GBS enhancement is considered to be related to inhomogeneous plastic deformation and is categorized as "slip-induced" GBS. The origin of the slip-induced GBS is attributed to plastic compatibility at grain boundaries under the presence of plastic strain anisotropy and the absorption and dissociation of lattice dislocations at grain boundaries.

\section{Acknowledgments}

This work was supported by a Grant-in-Aid for Scientific Research from the Ministry of Education, Culture, Sports, Science and Technology of Japan, "The Priority Group of Platform Science and Technology for Advanced Magnesium Alloys," Grant \# 11225202.

\section{REFERENCES}

1) W. F. Sheerly and R. R. Nash: Trans. Metall. Soc. AIME 218 (1960) 416.

2) T. Obara, H. Yoshinaga and S. Morozumi: Acta Metall. 21 (1973) 845.

3) H. Yoshinaga and R. Horiuchi: Trans. JIM 5 (1963) 14.

4) J. F. Stohr and J. P. Poirier: Philos. Mag. 5 (1972) 1313.

5) See for example, ASM Specialty Handbook, Magnesium and Magne- sium Alloys, ed. M. M. Avedesian and H. Baker, (ASM International, Materials Park, OH, 1999) p. 17.

6) T. Mukai, M. Yamanoi, H. Watanabe and K. Higashi: Scr. Mater. 45 (2001) 89.

7) J. Koike, T. Kobayashi, T. Mukai, H. Watanabe, M. Suzuki, K. Maruyama and K. Higashi: Acta Mater. 51 (2003) 2055.

8) F. E. Hauser, C. D. Starr, L. Tietz and J. E. Dorn: Trans. ASM 47 (1955) 102.

9) F. E. Hauser, P. R. Landon and J. E. Dorn: Trans. ASM 48 (1956) 986.

10) R. C. Gifkins and T. G. Langdon: J. Inst. Metals 93 (1964-1965) 347.

11) S. Hwang, C. Nishimura and P. G. McCormick: Scr. Mater. 44 (2001) 1507.

12) N. Wang, Z. Wang, K. T. Aust and U. Erb: Mater. Sci. Eng. A A237 (1997) 150.

13) M. Ke, S. A. Hackney, W. W. Miligan and E. C. Aifantis: Nanostruct. Mater. 5 (1995) 689

14) M. G. Zelin, H. S. Yang, R. Z. Valiev and A. K. Mekherjee: Metall. Trans. A 23A (1992) 3135.

15) R. L. Bell and T. G. Langdon: J. Mater. Sci. 2 (1967) 313.

16) T. G. Langdon and R. B. Vatava: Mechanical Testing for Deformation Model Development, ed. by R. W. Rohde and J. C. Swearengen (ASM STP 765, Materials Park, OH, 1982) p. 345.

17) P. R. Howell and G. L. Dunlop: Proc. 1st Int. Conf. Creep Fracture of Engineering Materials and Structures, ed. by B. Wilshire and D. R. J. Owen, (Pineridge Press, Swansea, 1981) p. 127.

18) V. M. Rosenberg and Epshtein: Fiz. Met. Metalloved. 9 (1960) 124.

19) J. A. Wlliams: Jernkont. Ann. 155 (1971) 343.

20) L. J. Slutsky and C. M. Garland: Phys. Rev. 107 (1957) 972.

21) H. K. Frost and M. F. Ashby: Deformation-Mechanism Maps, (Pergamon Press, Oxford, 1982) p. 44.

22) R. Z. Valiev and O. A. Kaibyshev: Acta Metall. 31 (1983) 2121.

23) P. Mussot, C. Rey and A. Zaoui: Res. Mechanica 14 (1985) 69.

24) A. E. H. Love: A Treatise on the Mathematical Theory of Elasticity, 4th ed., (Cambridge Univ. Press, republished by Dover, NY, 1944).

25) R. Z. Valiev and O. A. Kaibyshev: Phys. Status Solidi (a) 44 (1977) 477.

26) G. R. Kegg, C. A. P. Horton and J. M. Silcock: Philos. Mag. 27 (1973) 1041.

27) H. Kokawa, T. Watanabe and S. Karashima: Philos. Mag. A 44 (1981) 1239.

28) R. C. Pond: Proc. Roy. Soc. London A 357 (1977) 471.

29) J. Huber and M. Hatherly: Met. Sci. (1979) 665.

30) J. Huber and M. Hatherly: Z. Metallkde. 71 (1980) 15.

31) M. H. Yoo: Metall. Trans. A 12A (1981) 409. 